\title{
Quantitative determination and pattern recognition analyses of bioactive marker compounds from Dipsaci Radix by HPLC
}

\author{
Bing Tian Zhao · Su Yang Jeong • Dong Cheul Moon • \\ Kun Ho Son · Jong Keun Son • Mi Hee Woo
}

Received: 7 February 2013/Accepted: 22 May 2013/Published online: 23 July 2013

(c) The Author(s) 2013. This article is published with open access at Springerlink.com

\begin{abstract}
In this study, quantitative and pattern recognition analyses were developed using HPLC/UV for the quality evaluation of Dipsaci Radix. For quantitative analysis, five major bioactive compounds were assessed. The separation conditions employed for HPLC/UV were optimized using ODS $\mathrm{C}_{18}$ column $(250 \times 4.6 \mathrm{~mm}, 5 \mu \mathrm{m})$ with a gradient of acetonitrile and water as the mobile phase at a flow rate of $1.0 \mathrm{~mL} / \mathrm{min}$ and a detection wavelength of $212 \mathrm{~nm}$. These methods were fully validated with respect to linearity, accuracy, precision, recovery, and robustness. The HPLC/UV method was applied successfully to the quantification of five major compounds in the extract of Dipsaci Radix. The HPLC analytical method for pattern recognition analysis was validated by repeated analysis of 17 Dipsaci Radix and four Phlomidis Radix samples. The results indicate that the established HPLC/ UV method is suitable for quantitative analysis.
\end{abstract}

B. T. Zhao $\cdot$ S. Y. Jeong $\cdot$ M. H. Woo $(\bowtie)$

College of Pharmacy, Catholic University of Daegu, Gyeongsan 712-702, Korea

e-mail:woomh@cu.ac.kr

D. C. Moon

College of Pharmacy, Chungbuk National University, Cheongju, Korea

K. H. Son

College of Life Science, Andong National University, Andong, Korea

J. K. Son $(\bowtie)$

College of Pharmacy, Yeungnam University,

Gyeongsan 712-749, Korea

e-mail: jkson@yu.ac.kr
Keywords Dipsaci Radix - Dipsacus asperoides . HPLC-UV · Quality control

\section{Introduction}

The use of herbal medicines continues to expand rapidly throughout the world. Many people now take herbal medicines or herbal products for their health in different national health-care settings (WHO 2004). The requirements and methods for quality control of finished herbal products, particularly for mixed herbal products, are far more complex than for other pharmaceuticals. The quality of such products is influenced by the quality of the raw materials used. Good agricultural and good collection practices (GACP) for medicinal plants, including plant selection and cultivation, are therefore important measures (WHO 2003). Quality control in synthetic drugs is conducted by measuring their medicinal components, whereas quality control in herbal medicines is traditionally performed by measuring a representative compound (a marker compound) contained in the herbal medicines. However, quantitation of one or a few components is not an adequate approach for quality control of herbal medicines. Thus there is an urgent need to establish a comprehensive qualified evaluation method based on analysis of the bioactive compounds in order to accurately reflect the quality of herbal medicines. Fingerprint analysis/pattern recognition with multivariate statistical analysis can provide information regarding the overall chemical composition of herbal medicines, including the marker compounds traditionally used for quality control (Islam et al. 2009).

Dipsaci Radix is the roots of Dipsacus asperoides C. Y. Cheng et T. M. Ai (Dipsacaceae) in the Korean Herbal Pharmacopoeia (K. H. P.) (Korea Food and Drug 
Administration 2007) and the Chinese Pharmacopoeia (C. P.). Dipsaci Radix is controlled to contain $>2 \%$ of akebia saponin D in C. P. (Pharmacopoeia Commission of People's Republic of China 2010). Various saponins (Hung et al. 2005; Oh et al. 1999), iridoids (Tomita and Mouri 1996) and phenylpropanoids (Inoue et al. 1989) have been isolated from Dipsaci Radix. Biological studies have revealed that this plant possesses antioxidant (Hung et al. 2006), anti-inflammatory (Jung et al. 2012), anticomplementary (Oh et al. 1999), and cytotoxic effects (Tomita and Mouri 1996; Zhou et al. 2009).

Dipsaci Radix and Phlomidis Radix are different species. Phlomidis Radix is the roots of Phlomis umbrosa Turczaninow in the Korean Herbal Pharmacopoeia (K. H. P.) (Korea Food and Drug Administration 2007). However, Phlomidis Radix has often been misused as Dipsaci Radix in the Korean market because of similarities in their names and shapes. Therefore, we have selected Phlomidis Radix as a comparative herbal medicine. There is no report on the differentiation between Dipsaci Radix and Phlomidis Radix.

Some HPLC/UV analytical methods have been developed for the analysis of Dipsaci Radix and its related products. Ma et al. (2007) and Wang et al. (2006) reported akebia saponin D as a specific marker for the distinction of Dipsaci Radix. Those studies focused only on quantitative analysis of akebia saponin D, which is not a promising approach for the quality control or fingerprinting analysis of herbal drugs. However, as multiple compounds might be associated with therapeutic functions, a single marker compound could not be responsible for the overall pharmacological activities of Dipsaci Radix. Therefore, there is an urgent need to establish a comprehensive quality evaluation method based on analysis of a variety of active compounds in order to accurately reflect the quality of these herbal drugs. In the present study, a simple, sensitive and precise reverse-phase HPLC/UV method has been developed for the quantitative determination of five marker components, loganin (1), sweroside (2), dipsanoside A (3), 3 - $O$ - $[\beta$-D-glu- $(1 \rightarrow 4)][\alpha$-L-rha- $(1 \rightarrow 3)]-\beta$-D-glu $(1 \rightarrow 3)-\alpha$-Lrha- $(1 \rightarrow 2)-\alpha$-L-ara-hed $28-O-\beta$-D-glu- $(1 \rightarrow 6)-\beta$-D-glu ester (4) and akebia saponin D (5), along with a pattern-recognition method for the quality control of Dipsaci Radix extract. Using this method, the contents of bioactive compounds in seventeen Dipsaci Radix and four Phlomidis Radix samples from China and Korea were analyzed and compared.

Using this method, the contents of bioactive compounds in seventeen Dipsaci Radix from China and four Phlomidis Radix from Korea samples were analyzed and compared. We used all Dipsaci Radix samples from China, because its Korean samples couldn't be available in the herbal markets. The PAM method of pattern analysis was subsequently applied to the quality control of the roots of Dipsaci Radix and Phlomidis Radix.

\section{Materials and methods}

Plant materials

Twenty-one samples including seventeen Dipsaci Radix and four Phlomidis Radix samples cultivated in different regions were provided by the National Center for Standardization of Herbal Medicine. Dipsaci Radix (D01-D17) and Phlomidis Radix (P18-P21) included D01 (Si Chuan Sheng, China), D02 (Ji Zhou, China), D03 (unknown area, China), D04 (unknown area, China), D05 (unknown area, China), D06 (unknown area), D07 (unknown area), D08 (unknown area), D09 (unknown area, China), D10 (unknown area), D11 (An Guo, China), D12 (Si Chuan Sheng, China, processed with salt-water), D13 (unknown area, processed with salt-water), D14 (unknown area, processed with wine), D15 (Si Chuan Sheng, China, processed with wine), D16 (Nan Zhou, China), D17 (unknown area, China), P18 (Yeong Cheon, Korea), P19 (Je Chon, Korea), P20 (Yeong Cheon, Korea) and P21 (Yeong Cheon, Korea).

\section{Reagents}

All of the standard compounds were provided by Prof. Kun Ho Son, Andong National University, Andong, Korea. Their structures were unambiguously identified as loganin (1), sweroside (2), dipsanoside A (3), 3- $O$-[ $\beta$-D-glu$(1 \rightarrow 4)][\alpha$-L-rha- $(1 \rightarrow 3)]-\beta$-D-glu $(1 \rightarrow 3)-\alpha$-L-rha- $(1 \rightarrow 2)-\alpha$-Lara-hed $28-O$ - $\beta$-D-glu-( $1 \rightarrow 6)-\beta$-D-glu ester (4) and akebia saponin D (5) based on NMR and MS data compared with published data. The standard compound structures are shown in Fig. 1. Purity of standard compounds was estimated to be higher than $95 \%$ based on HPLC and LC-MS/ MS analysis. Internal standard (I.S.), pulsatilla saponin $\mathrm{H}$, was provided as powder from Prof. Sam Sik Kang, Seoul National University. HPLC-grade methanol and acetonitrile were purchased from Merck K GaA (Darmstadt, Germany). All other chemicals used were of analytical grade unless otherwise noted. Distilled water was prepared using Milli-Q purification system (Millipore, Bedford, MA, USA).

\section{Sample preparation}

Dried rhizome powder was used to determine the contents of the five marker compounds and pattern recognition analysis of each extraction of Dipsaci Radix. Powdered Dipsaci Radix was sieved through 50 mesh, and about 
<smiles>COC(=O)C1C=COC(OC)[C@H]1[C@H]1C[C@H](O)C(C)[C@H]1C(=O)O</smiles>

1. loganin<smiles>C=CC1C(OC)OC=C2C(=O)OCCC21</smiles>

2. sweroside

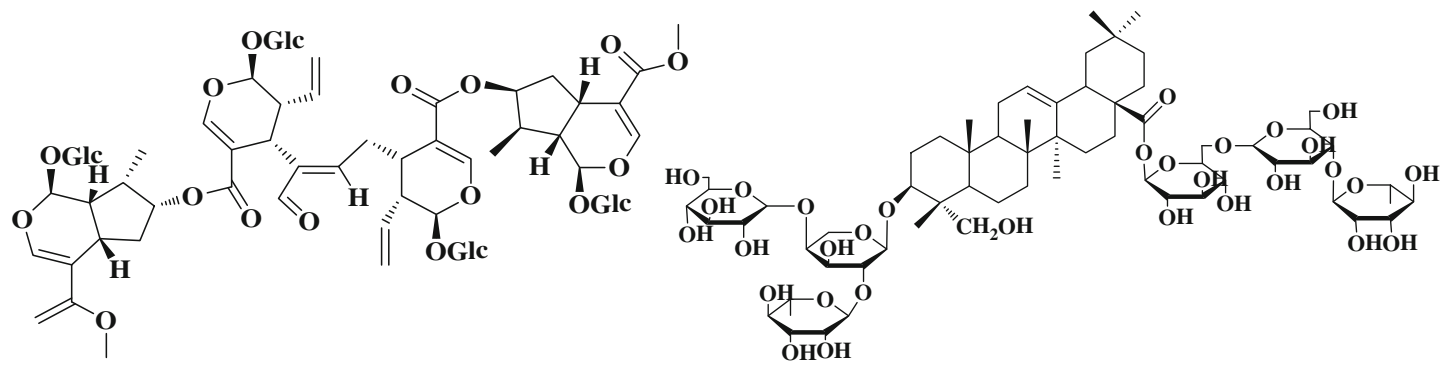

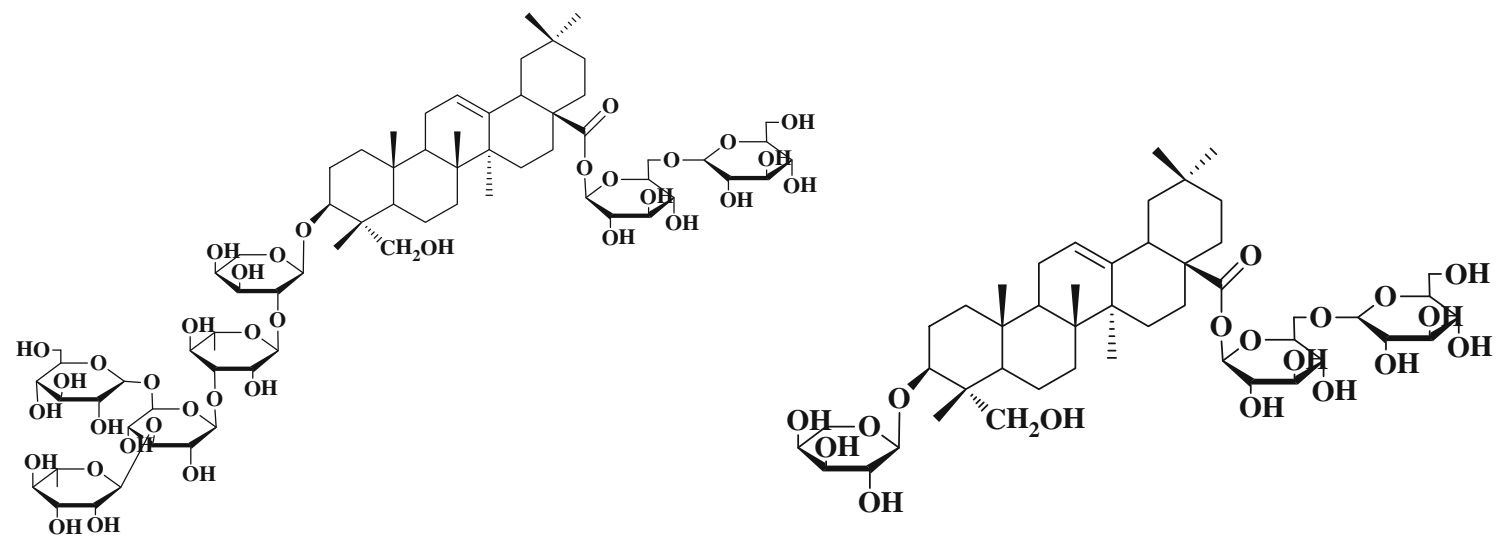

4. 3- $O-[\beta$-D-glu- $(1 \rightarrow 4)][\alpha-\mathrm{L}-$ rha$(1 \rightarrow 3)]-\beta$-D-glu $(1 \rightarrow 3)-\alpha$-L-rha$(1 \rightarrow 2)-\alpha-$-ara-hed $28-O-\beta$-D-glu$(1 \rightarrow 6)-\beta$-D-glu ester

Fig. 1 Chemical structures of standards

$0.5 \mathrm{~g}$ of the powder was accurately weighed; $25 \mathrm{~mL}$ of $50 \%$ methanol were added, the weight was accurately measured, and the sample was sonicated for $30 \mathrm{~min}$. The solution was weighed again, and the loss in weight was made up with $50 \%$ methanol. The solution was filtered through a $0.45-\mu \mathrm{m}$ membrane filter and the filtrate was used as the test solution. Sample solution of $20 \mu \mathrm{L}$ was injected into the HPLC system.

\section{HPLC/UV conditions}

The HPLC equipment was a Waters HPLC system (Waters, Milford, MA, USA) with a Waters 600 pumps, a Waters 486 UV detector and a Waters 717 autosampler. YMC ODS-H80 $(250 \times 4.6 \mathrm{~mm}, 4 \mu \mathrm{m})$, Shiseido capcell pak $(250 \times 4.6 \mathrm{~mm}, 5 \mu \mathrm{m})$ and Shodex ODS pak $(250 \times 4.6 \mathrm{~mm}$, $5 \mu \mathrm{m})$ columns were tested with the guard column filled with 
the same stationary phase. A (100\% acetonitrile) and B (water) were used as the mobile phase under a gradient condition (0 min, $15 \%$ A; 60 min, $35 \%$ A). The mobile phase was filtered under vacuum through a $0.45-\mu \mathrm{m}$ membrane filter and degassed prior to use. The analysis was carried out at a flow rate of $1.0 \mathrm{~mL} / \mathrm{min}$ with the detection wavelength set to $212 \mathrm{~nm}$, and the total run time was $60 \mathrm{~min}$. All compounds could be resolved with baseline separation at $212 \mathrm{~nm}$ with maximum absorption. Hence, characteristic chromatographic patterns were obtained at $212 \mathrm{~nm}$. The chromatograms were processed using Empower Pro software, Build 1154 (Waters, Milford, MA).

\section{Analytical method validation}

The standards (4 mg) of loganin (1), sweroside (2), dipsanoside A $(3), 3-O$ - $[\beta$-D-glu- $(1 \rightarrow 4)][\alpha$-L-rha- $(1 \rightarrow 3)]$ $\beta$-D-glu $(1 \rightarrow 3)$ - $\alpha$-L-rha- $(1 \rightarrow 2)$ - $\alpha$-L-ara-hed $\quad 28-O$ - $\beta$-D-glu$(1 \rightarrow 6)$ - $\beta$-D-glu ester (4) and akebia saponin D (5) were each accurately weighed and then dissolved in $10 \mathrm{~mL}$ of $100 \%$ methanol to produce stock standard solutions of $400 \mathrm{ppm}$. The internal standard (pulsatilla saponin $\mathrm{H}$; $15 \mathrm{mg}$ ) was accurately weighed and then dissolved in $10 \mathrm{~mL}$ of $100 \%$ methanol to produce a stock solution of $1,500 \mathrm{ppm}$. The calibration curves were made by diluting the stock solutions with $100 \%$ methanol. A reference solution of the five standard compounds at concentrations of $0.1-200 \mu \mathrm{g} / \mathrm{mL}$ was analyzed by HPLC/UV. The regression equations were calculated in the form of $y=a x+b$, where $y$ and $x$ correspond to peak area ratio for internal standard and compound concentration, respectively. Recovery tests were executed by mixing a powdered sample $(0.5 \mathrm{~g})$ with the reference compounds at three control levels (near the LOQ, medium and higher concentrations for calibration curve of each compound contained in the samples). The mixture was then extracted by sonication in $25 \mathrm{~mL}$ of $50 \%$ methanol for $30 \mathrm{~min}$. The extract solution was filtered through a $0.45-\mu \mathrm{m}$ membrane. The HPLC/UV analysis experiments were performed in triplicate for each control level. The data from the standard solution and the extracted sample were compared. Precision and accuracy were determined by multiple analyses ( $n=5$ ) of quality control samples prepared at low, medium and high concentrations spanning the calibration range.

\section{Pattern recognition analysis}

To evaluate the phytochemical equivalency among the 21 samples comprising seventeen Dipsaci Radix and four Phlomidis Radix samples, pattern recognition analysis was conducted. In this study we used three marker compound peaks [sweroside (2), dipsanoside A (3) and akebia saponin $\mathrm{D}(\mathbf{5})$ ] for pattern recognition analysis. Pattern recognition analysis was conducted using software package R-2.11.0.

\section{Results and discussion}

Optimization of chromatographic conditions

The HPLC conditions were selected according to the requirement for obtaining chromatograms with better resolution of adjacent peaks within a short retention time. For the optimization of chromatographic conditions, the effect of the mobile phase composition on the separation was examined. A mobile phase of water-methanol did not result in satisfactory separation of structurally similar compounds. Acetonitrile as an organic modifier significantly improved the separation. We also tested the addition of $0.1,1$ and $10 \%$ acid (acetic acid, formic acid and phosphoric acid) in the water. The water without acid resulted in good resolution of all compounds, as well as satisfactory peak symmetry and shape. The typical chromatograms of samples and standard mixture are shown in Fig. 2, which shows that all target compounds and an internal standard are completely separated within $60 \mathrm{~min}$. Pulsatilla saponin H (I.S.) was selected as an internal standard. The chromatographic peaks of the analytes in sample solution were identified by comparing their retention times with those of the reference standards and were further confirmed by spiking samples with reference compounds (Fig. 2). All compounds could be resolved with baseline separation at $212 \mathrm{~nm}$ with the maximum absorption shown for the five constituents. Hence, characteristic chromatographic patterns were obtained at $212 \mathrm{~nm}$.

\section{Optimization of sample preparation conditions}

Eight extracting solvents, $100 \%$ ethanol, $75 \%$ ethanol, $50 \%$ ethanol, $25 \%$ ethanol, $100 \%$ methanol, $75 \%$ methanol, $50 \%$ methanol and $25 \%$ methanol, were compared in sample assays after sonication for $30 \mathrm{~min}$. When the sample was extracted with $50 \%$ methanol, the sample assay was higher than the other solvent samples. Therefore, we employed $50 \%$ methanol as the extracting solvent throughout this work. Two extraction methods, ultra-sonication and reflux using $50 \%$ methanol as an extraction solvent, were compared in sample assays. The sample assay results after the sonication extraction method were higher than those after reflux. To determine the time needed for complete extraction, samples were extracted for five different lengths of time (10, 20, 30, 40 and $60 \mathrm{~min})$. Thus $50 \%$ methanol solvent and the sonication extraction method were employed. When the extraction time was 
Fig. 2 HPLC chromatograms of standard mixture (a), Dipsaci Radix (b) and Phlomidis Radix (c). 1 loganin, 2 sweroside, 3 dipsanoside A, 4 3- $O$-[ $\beta$-D-glu$(1 \rightarrow 4)][\alpha$-L-rha- $(1 \rightarrow 3)]-\beta$-D$\operatorname{glu}(1 \rightarrow 3)-\alpha$-L-rha- $(1 \rightarrow 2)-\alpha$-Lara-hed $28-O-\beta$-D-glu- $(1 \rightarrow 6)-\beta$ D-glu ester, 5 akebia saponin D, I.S. pulsatilla saponin $\mathrm{H}$

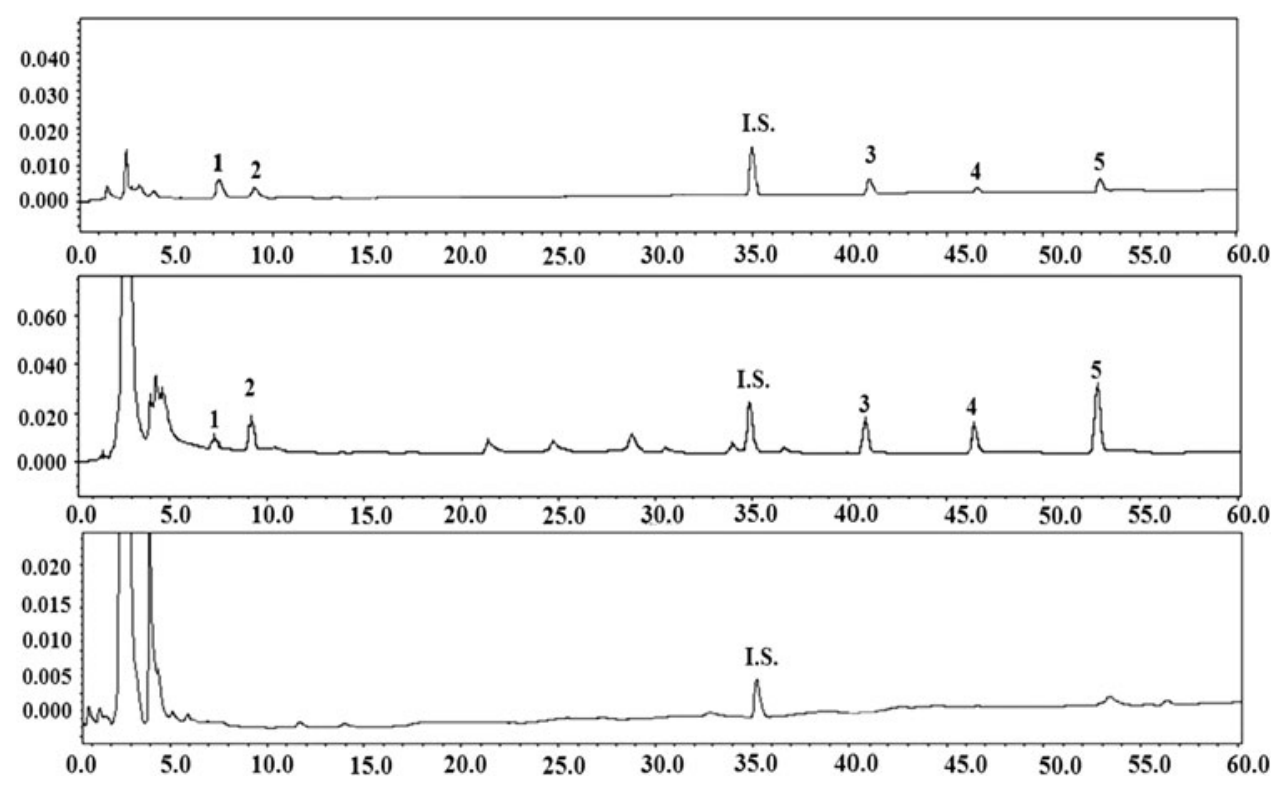

$30 \mathrm{~min}$, the sample assay results were similar to those at $40 \mathrm{~min}$. Therefore, when the extraction time was $30 \mathrm{~min}$, all of the compounds were sufficiently extracted.

Validation of the method

\section{Linearity, calibration range, limits of detection and quantification}

Each coefficient of correlation $\left(r^{2}\right)$ was $>0.999$, as determined by least square analysis, suggesting good linearity between the peak area ratio and the compound concentrations (Table 1). The limits of detection (LOD) and limits of quantitation (LOQ) were evaluated based on the lowest detectable peak in the chromatogram having a signal-to-noise $(\mathrm{S} / \mathrm{N})$ ratio of 3 and 10 , respectively. The LOD and LOQ under our experimental conditions are listed in Table 1. The obtained values for both LOD and LOQ for these five standards were low enough to detect traces of these compounds in either crude extract or its preparation.

\section{Precision and accuracy}

The extraction recovery test was performed by extracting a known amount of the five compounds from the Dipsaci Radix powder samples. Known amounts of each standard compound at three levels were mixed with the sample powder and extracted with $50 \%$ methanol, as described in the experimental section. The \% recovery of each standard ranged from 97.98 to $105.12 \%$, and the RSD was $<1.44 \%$ (Table 2). The average recovery was represented by the formula: $\mathrm{R}(\%)=[$ (amount from the sample spiked standard - amount from the sample)/amount from the spiked standard] $\times 100$.

Intra-assay precision and accuracy were determined from the variability of multiple analyses $(n=5)$ of quality control samples analyzed within the same analytical run. The quality control samples had intra-assay precision below $0.50 \%$ and accuracy between 96.67 and $102.49 \%$. Inter-assay precision and accuracy were evaluated from the variability of multiple analyses $(n=5)$ of quality control samples analyzed on a single analytical run for consecutive 5 days. The quality control samples had an inter-assay
Table 1 Linearity, linear ranges, LOD and LOQ

1 loganin, 2 sweroside, 3 dipsanoside A, 4 3- $O$-[ $\beta$-D-glu$(1 \rightarrow 4)][\alpha$-L-rha- $(1 \rightarrow 3)]-\beta$-D$\operatorname{glu}(1 \rightarrow 3)-\alpha$-L-rha- $(1 \rightarrow 2)-\alpha$-Lara-hed $28-O-\beta$-D-glu- $(1 \rightarrow 6)-\beta$ D-glu ester, 5 akebia saponin D

\begin{tabular}{lllllll}
\hline Analytes & $\begin{array}{l}\text { Linear range } \\
(\mu \mathrm{g} / \mathrm{mL})\end{array}$ & $\begin{array}{l}\text { Slope } \\
(\mathrm{a})\end{array}$ & $\begin{array}{l}\text { Intercept } \\
(\mathrm{b})\end{array}$ & $\begin{array}{l}\text { Correlation } \\
\text { coefficient }\left(r^{2}\right)\end{array}$ & $\begin{array}{l}\text { LOD } \\
(\mathrm{ng} / \mathrm{mL})\end{array}$ & $\begin{array}{l}\text { LOQ } \\
(\mathrm{ng} / \mathrm{mL})\end{array}$ \\
\hline $\mathbf{1}$ & $0.1-40$ & 0.079 & 0.0318 & 0.9998 & 120 & 350 \\
$\mathbf{2}$ & $0.1-40$ & 0.054 & -0.0120 & 0.9999 & 140 & 320 \\
$\mathbf{3}$ & $0.1-50$ & 0.1745 & -0.0057 & 0.9999 & 100 & 290 \\
$\mathbf{4}$ & $0.1-100$ & 0.0389 & -0.0508 & 0.9998 & 130 & 270 \\
$\mathbf{5}$ & $0.1-200$ & 0.0376 & -0.0522 & 0.9999 & 120 & 330 \\
\hline
\end{tabular}


Table 2 Recoveries of marker compounds through standard addition $(\mathrm{n}=6)$
1 loganin, 2 sweroside, 3

dipsanoside A, 4 3- $O$ - $[\beta$-D-glu$(1 \rightarrow 4)][\alpha$-L-rha- $(1 \rightarrow 3)]-\beta$-D$\operatorname{glu}(1 \rightarrow 3)$ - $\alpha$-L-rha- $(1 \rightarrow 2)-\alpha$-Lara-hed $28-O-\beta$-D-glu- $(1 \rightarrow 6)-\beta$ D-glu ester, 5 akebia saponin D

${ }^{a}$ Relative standard deviation

\begin{tabular}{|c|c|c|c|c|c|}
\hline Analytes & $\begin{array}{l}\text { Fortified conc. } \\
(\mu \mathrm{g} / \mathrm{mL})\end{array}$ & $\begin{array}{l}\text { Observed conc. } \\
(\mu \mathrm{g} / \mathrm{mL})\end{array}$ & $\begin{array}{l}\mathrm{RSD}^{\mathrm{a}} \\
(\%)\end{array}$ & $\begin{array}{l}\text { Calculated } \\
\text { recovery }(\%)\end{array}$ & $\begin{array}{l}\text { Mean } \\
\text { recovery }(\%)\end{array}$ \\
\hline \multirow[t]{4}{*}{1} & 0.0 & 8.47 & - & - & \multirow[t]{4}{*}{105.12} \\
\hline & 0.4 & 8.92 & 0.30 & 112.50 & \\
\hline & 20.0 & 28.89 & 0.38 & 102.10 & \\
\hline & 40.0 & 48.77 & 0.06 & 100.75 & \\
\hline \multirow[t]{4}{*}{2} & 0.0 & 51.29 & - & - & \multirow[t]{4}{*}{99.35} \\
\hline & 0.4 & 51.26 & 0.20 & 92.50 & \\
\hline & 20.0 & 72.15 & 0.11 & 104.30 & \\
\hline & 40.0 & 91.79 & 0.04 & 101.25 & \\
\hline \multirow[t]{4}{*}{3} & 0.0 & 17.38 & - & - & \multirow[t]{4}{*}{101.49} \\
\hline & 0.3 & 17.69 & 0.50 & 103.33 & \\
\hline & 20.0 & 37.67 & 0.04 & 101.45 & \\
\hline & 50.0 & 67.22 & 0.02 & 99.68 & \\
\hline \multirow[t]{4}{*}{4} & 0.0 & 80.13 & - & - & \multirow[t]{4}{*}{97.98} \\
\hline & 0.3 & 80.42 & 0.04 & 96.67 & \\
\hline & 50.0 & 128.20 & 0.02 & 96.14 & \\
\hline & 100.0 & 181.26 & 0.01 & 101.13 & \\
\hline \multirow[t]{4}{*}{5} & 0.0 & 111.27 & - & - & \multirow[t]{4}{*}{101.72} \\
\hline & 0.3 & 111.58 & 0.07 & 103.33 & \\
\hline & 100.0 & 211.5 & 0.01 & 100.23 & \\
\hline & 200.0 & 314.48 & 0.01 & 101.61 & \\
\hline
\end{tabular}

Table 3 Precision and accuracy of analytical results $(n=5)$

\begin{tabular}{|c|c|c|c|c|c|c|c|c|c|}
\hline \multirow[t]{2}{*}{ Analytes } & \multirow{2}{*}{$\begin{array}{l}\text { Nominal } \\
\text { conc. }^{\mathrm{a}}(\mu \mathrm{g} / \mathrm{mL})\end{array}$} & \multicolumn{4}{|c|}{ Intra-day $(n=5)$} & \multicolumn{4}{|c|}{ Inter-day $(\mathrm{n}=5)$} \\
\hline & & $\begin{array}{l}\text { Observed }^{\mathrm{b}} \\
(\mu \mathrm{g} / \mathrm{mL})\end{array}$ & S.D. ${ }^{\mathrm{c}}$ & $\begin{array}{l}\text { Accuracy }^{\mathrm{d}} \\
(\%)\end{array}$ & Precision $^{\mathrm{e}}$ & $\begin{array}{l}\text { Observed } \\
(\mu \mathrm{g} / \mathrm{mL})\end{array}$ & S.D. & $\begin{array}{l}\text { Accuracy } \\
(\%)\end{array}$ & Precision \\
\hline \multirow[t]{3}{*}{1} & 0.4 & 0.41 & 0.01 & 102.49 & 0.30 & 0.41 & 0.02 & 102.50 & 1.06 \\
\hline & 20.0 & 20.09 & 0.01 & 100.45 & 0.38 & 20.03 & 0.08 & 100.15 & 1.62 \\
\hline & 40.0 & 40.04 & 0.01 & 100.10 & 0.06 & 40.05 & 0.14 & 100.13 & 1.40 \\
\hline \multirow[t]{3}{*}{2} & 0.4 & 0.39 & 0.02 & 97.50 & 0.20 & 0.39 & 0.06 & 97.50 & 0.58 \\
\hline & 20.0 & 19.78 & 0.02 & 98.91 & 0.11 & 20.12 & 0.47 & 100.60 & 2.42 \\
\hline & 40.0 & 40.01 & 0.02 & 100.02 & 0.04 & 40.19 & 0.91 & 100.48 & 2.34 \\
\hline \multirow[t]{3}{*}{3} & 0.3 & 0.31 & 0.02 & 96.67 & 0.50 & 0.31 & 0.10 & 103.33 & 2.08 \\
\hline & 20.0 & 20.07 & 0.03 & 100.34 & 0.04 & 20.02 & 0.13 & 100.10 & 1.26 \\
\hline & 50.0 & 49.78 & 0.01 & 99.57 & 0.02 & 50.06 & 0.36 & 100.12 & 1.79 \\
\hline \multirow[t]{3}{*}{4} & 0.3 & 0.29 & 0.01 & 96.67 & 0.04 & 0.30 & 0.42 & 100.00 & 2.11 \\
\hline & 50.0 & 49.72 & 0.02 & 99.44 & 0.02 & 49.98 & 0.49 & 99.96 & 1.21 \\
\hline & 100.0 & 99.92 & 0.02 & 99.92 & 0.01 & 100.36 & 0.93 & 100.36 & 1.71 \\
\hline \multirow[t]{3}{*}{5} & 0.3 & 0.29 & 0.02 & 96.67 & 0.07 & 0.30 & 0.35 & 100.00 & 1.40 \\
\hline & 100.0 & 99.88 & 0.01 & 99.88 & 0.01 & 100.04 & 0.55 & 100.04 & 1.10 \\
\hline & 200.0 & 200.09 & 0.01 & 100.05 & 0.01 & 200.12 & 0.61 & 100.06 & 0.62 \\
\hline
\end{tabular}

a Added concentration of standard

b Amount from the sample spiked standard - amount from the sample

c Standard deviation

d (Observed/added) $\times 100$

e The relative standard deviation of accuracy

1 loganin, 2 sweroside, 3 dipsanoside A, 4 3- $O$-[ $\beta$-D-glu- $(1 \rightarrow 4)][\alpha$-L-rha- $(1 \rightarrow 3)]-\beta$-D-glu( $(1 \rightarrow 3)$ - $\alpha$-L-rha- $(1 \rightarrow 2)-\alpha$-L-ara-hed $28-O$ - $\beta$-D-glu$(1 \rightarrow 6)$ - $\beta$-D-glu ester, 5 akebia saponin D 
Table 4 Contents (wt $\%$ ) of five components in Dipsaci Radix (D01-D17) and Phlomidis Radix (P18-P21) samples a Each value represents the mean \pm S.D. $(n=3)$

$\mathrm{b}$ The average contents of all the Dipsaci Radix (D01-D17)

\begin{tabular}{|c|c|c|c|c|c|}
\hline \multirow[t]{2}{*}{ Sample } & \multicolumn{5}{|c|}{ Contents (w/w \%) } \\
\hline & 1 & 2 & 3 & 4 & 5 \\
\hline D01 & $0.06 \pm 0.01^{\mathrm{a}}$ & $0.98 \pm 0.01$ & $0.29 \pm 0.01$ & $1.67 \pm 0.06$ & $10.62 \pm 0.52$ \\
\hline D02 & $0.01 \pm 0.00$ & $0.28 \pm 0.00$ & $0.15 \pm 0.01$ & $1.60 \pm 0.06$ & $3.61 \pm 0.09$ \\
\hline D03 & $0.22 \pm 0.01$ & $0.87 \pm 0.02$ & $0.28 \pm 0.01$ & $0.88 \pm 0.01$ & $10.81 \pm 0.29$ \\
\hline D04 & $0.08 \pm 0.01$ & $0.75 \pm 0.00$ & $0.24 \pm 0.01$ & $1.70 \pm 0.03$ & $10.96 \pm 0.10$ \\
\hline D05 & $0.01 \pm 0.00$ & $0.52 \pm 0.02$ & $0.39 \pm 0.01$ & $1.05 \pm 0.02$ & $6.41 \pm 0.16$ \\
\hline D06 & $0.05 \pm 0.01$ & $0.73 \pm 0.03$ & $0.24 \pm 0.02$ & $1.53 \pm 0.02$ & $8.14 \pm 0.13$ \\
\hline D07 & $0.07 \pm 0.01$ & $0.42 \pm 0.01$ & $0.27 \pm 0.01$ & $0.83 \pm 0.03$ & $4.80 \pm 0.05$ \\
\hline D08 & $0.04 \pm 0.01$ & $0.40 \pm 0.02$ & $0.20 \pm 0.01$ & $0.84 \pm 0.01$ & $5.05 \pm 0.06$ \\
\hline D09 & $0.05 \pm 0.01$ & $0.73 \pm 0.01$ & $0.35 \pm 0.01$ & $1.26 \pm 0.03$ & $9.18 \pm 0.30$ \\
\hline D10 & $0.07 \pm 0.01$ & $0.70 \pm 0.01$ & $0.36 \pm 0.01$ & $1.21 \pm 0.02$ & $4.00 \pm 0.16$ \\
\hline D11 & $0.11 \pm 0.01$ & $0.28 \pm 0.01$ & $0.14 \pm 0.00$ & $0.44 \pm 0.01$ & $3.71 \pm 0.10$ \\
\hline D12 & $0.06 \pm 0.01$ & $0.64 \pm 0.02$ & $0.28 \pm 0.00$ & $1.28 \pm 0.04$ & $1.70 \pm 0.01$ \\
\hline D13 & $0.16 \pm 0.01$ & $0.38 \pm 0.00$ & $0.01 \pm 0.00$ & $0.01 \pm 0.00$ & $0.73 \pm 0.02$ \\
\hline D14 & $0.38 \pm 0.01$ & $0.70 \pm 0.02$ & $0.08 \pm 0.01$ & $0.44 \pm 0.02$ & $3.16 \pm 0.06$ \\
\hline D15 & $0.15 \pm 0.01$ & $0.87 \pm 0.03$ & $0.13 \pm 0.01$ & $0.55 \pm 0.01$ & $9.16 \pm 0.14$ \\
\hline D16 & $0.05 \pm 0.01$ & $0.55 \pm 0.02$ & $0.22 \pm 0.01$ & $1.36 \pm 0.06$ & $7.30 \pm 0.24$ \\
\hline D17 & $0.01 \pm 0.00$ & $0.32 \pm 0.00$ & $0.18 \pm 0.01$ & $0.98 \pm 0.03$ & $5.45 \pm 0.15$ \\
\hline Average $^{\mathrm{b}}$ & $0.09 \pm 0.00$ & $0.60 \pm 0.01$ & $0.22 \pm 0.01$ & $1.04 \pm 0.04$ & $6.16 \pm 0.12$ \\
\hline P18 & - & - & - & - & - \\
\hline P19 & - & - & - & - & - \\
\hline $\mathrm{P} 20$ & - & - & - & - & - \\
\hline $\mathrm{P} 21$ & - & - & - & - & - \\
\hline
\end{tabular}

precision of $<2.42 \%$ and accuracy between 97.50 and $103.33 \%$. Thus, the developed method is highly reproducible; the precision and accuracy data are presented in Table 3.

\section{Robustness}

The robustness was determined in order to evaluate the reliability of the established HPLC method. All of the parameters were maintained so there would not be any interference with other peaks for the Dipsaci Radix. The experimental conditions, such as column temperature, column species and flow rate, were purposely altered. The theoretical plate $(N)$, capacity factor $\left(k^{\prime}\right)$, separation factor $(\alpha)$ and resolution $(R s)$ were evaluated. To evaluate their suitability, three different columns, YMC, Phenomenex and Shodex, were compared with regard to four analytical factors $\left(N, k^{\prime}, \alpha\right.$ and $\left.R \mathrm{~s}\right)$ on the column temperature of $30{ }^{\circ} \mathrm{C}$. The result showed that the four analytical factors did not differ greatly among the column species. Four different column temperatures, $25,30,35$ and $40{ }^{\circ} \mathrm{C}$, were compared with regard to these four analytical factors using the YMC column. Again the four analytical factors did not differ greatly by column temperature. Three different flow rates, $0.9,1.0$ and $1.1 \mathrm{~mL} / \mathrm{min}$, were also compared with regard to the four analytical factors using the YMC column at $30{ }^{\circ} \mathrm{C}$. The four analytical factors did not differ greatly by flow rate. We sought to optimize the chromatographic parameters, but the four analytical factors did not differ greatly when the conditions were changed; therefore these experimental conditions were sufficiently robust.

The sample stability was tested with a standard mixture solution at $0,0.5,1,2,5,10,15$ and 30 days. During this period, the solution was stored in the dark at room temperature or at $4{ }^{\circ} \mathrm{C}$. The resulting data indicated that all marker analytes remained stable (99.99\%) during the experimental period.

\section{Sample analysis}

The developed HPLC/UV method was then applied to the simultaneous determination of the five compounds, loganin (1), sweroside (2), dipsanoside A (3), 3- $O$-[ $\beta$-D-glu$(1 \rightarrow 4)][\alpha$-L-rha- $(1 \rightarrow 3)]$ - $\beta$-D-glu $(1 \rightarrow 3)$ - $\alpha$-L-rha- $(1 \rightarrow 2)-\alpha$-Lara-hed $28-O$ - $\beta$-D-glu- $(1 \rightarrow 6)-\beta$-D-glu ester $(4)$ and akebia saponin D (5) in Dipsaci Radix and Phlomidis Radix. The quantity of each compound present in samples was determined and the results are summarized in Table 4. Each sample was analyzed in triplicate to ensure the reproducibility of the quantitative result. Loganin (0.01-0.38\%), 


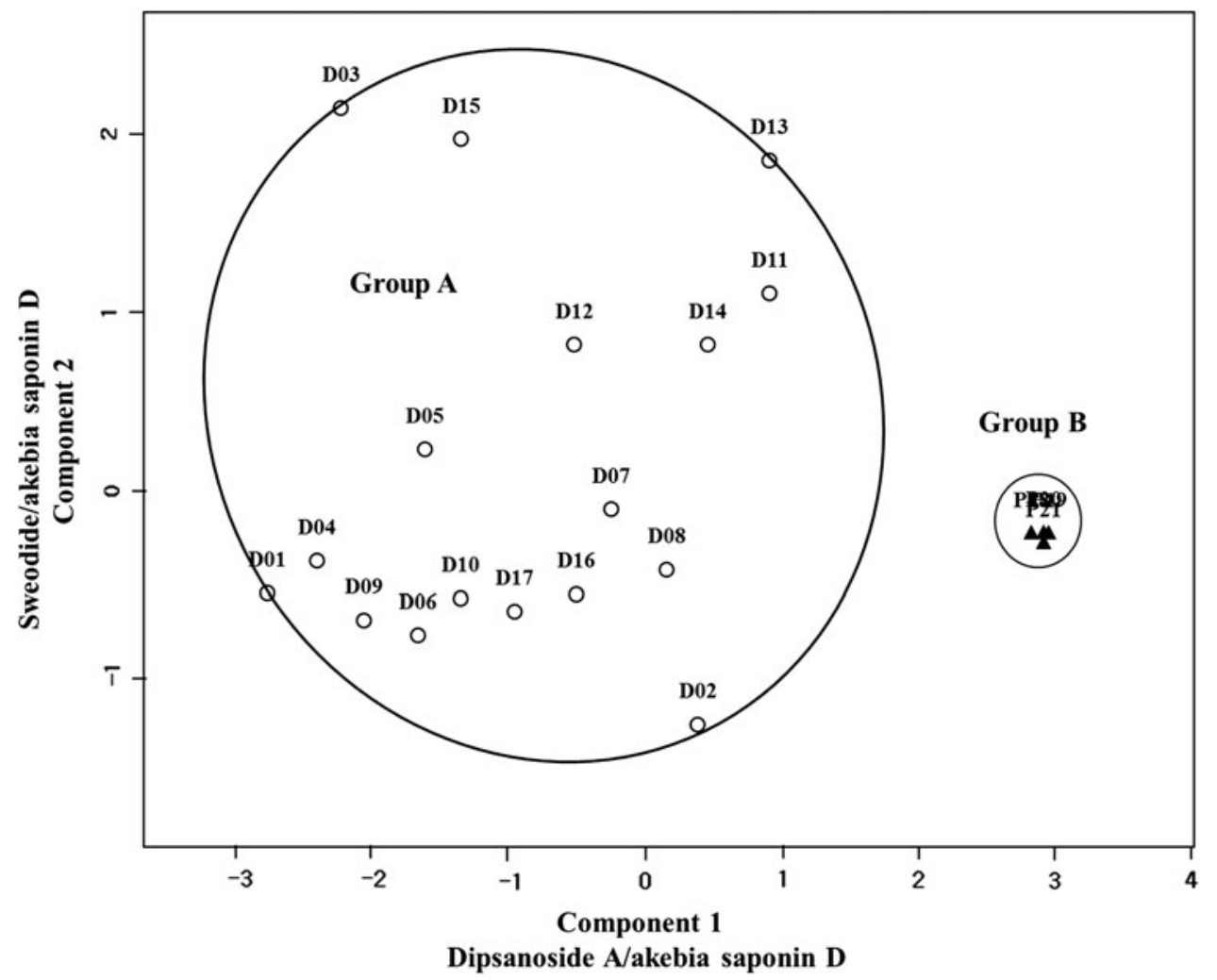

Fig. 3 PAM of Dipsaci Radix (A: D01-D17) and Phlomidis Radix (B: P18-P21)

sweroside $(0.28-0.98 \%)$, dipsanoside A (0.01-0.39\%), 3- $O$ - $[\beta$-D-glu- $(1 \rightarrow 4)][\alpha$-L-rha- $(1 \rightarrow 3)]-\beta$-D-glu( $1 \rightarrow 3)-\alpha-$ L-rha- $(1 \rightarrow 2)-\alpha$-L-ara-hed $28-O-\beta$-D-glu- $(1 \rightarrow 6)-\beta$-D-glu ester $(0.01-1.70 \%)$ and akebia saponin D (0.73-10.96\%) were found in Dipsaci Radix. These Dipsaci Radix components clustered into one group, and the most abundant component was akebia saponin D (0.73-10.96\%). Contents of akebia saponin D in D12 and D13 for salt-water processing were 1.70 and $0.73 \%$, respectively. Neither D12 nor D13 was suitable based on the regulation of $>2 \%$ in the Chinese Pharmacopoeia [5]. Thus, the major compounds in Dipsaci Radix appeared to change due to salt-water processing. In contrast, compounds $\mathbf{1 - 5}$ were not completely contained in Phlomidis Radix as a comparison herbal medicine. In the quantitative analysis, Dipsaci Radix and Phlomidis Radix samples clustered into two groups as mentioned below.

\section{Pattern recognition analysis}

To evaluate the phytochemical equivalency between 17 Dipsaci Radix and four Phlomidis Radix samples, pattern recognition analysis was conducted. In this study we used three marker compound peaks [sweroside (2), dipsanoside A (3) and akebia saponin D (5)] for pattern recognition analysis. Even though the content of compound $\mathbf{4}$ in
Dipsaci Radix was higher than those of compounds $\mathbf{2}$ and $\mathbf{3}$, we selected $\mathbf{2}, \mathbf{3}$ and $\mathbf{5}$ as marker compounds rather than 4 because of difficulties in the isolation and the availability of 4. From the pattern analysis of Partitioning Around Medoids (PAM) analyses (Fig. 3), all of the samples were clustered into two groups: A (D01-D17, Dipsaci Radix) and B (P18-P21, Phlomidis Radix).

Acknowledgments This research was supported by a grant (09112KFDA817 and 12172KFDA989) from the National Center for Standardization of Herbal Medicine funded by the Korea Food Drug Administration, Republic of Korea (2009).

Open Access This article is distributed under the terms of the Creative Commons Attribution License which permits any use, distribution, and reproduction in any medium, provided the original author(s) and the source are credited.

\section{References}

Hung, T.M., W.Y. Jin, P.T. Thuong, K.S. Song, Y.H. Seong, and K.H. Bae. 2005. Cytotoxic saponins from the root of Dipsacus asper wall. Archives of Pharmacal Research 28: 1053-1056.

Hung, T.M., M.K. Na, P.T. Thuong, N.D. Su, D.E. Sok, K.S. Song, Y.H. Seong, and K.H. Bae. 2006. Antioxidant activity of caffeoyl quinic acid derivatives from the roots of Dipsacus asper wall. Journal of Ethnopharmacology 108: 188-192. 
Inoue, K., T. Tanahashi, H. Inouye, H. Kuwajima, and K. Takaishi. 1989. Intermediacy of 6-hydroxyloganin in the ring cleavage course of loganin to secologanin. Phytochemistry 28: 2971-2979.

Islam, M.N., S.K. Lee, S.Y. Jeong, D.H. Kim, C.B. Jin, and H.H. Yoo. 2009. Quantitative and pattern recognition analyses for the quality evaluation of Herba Epimedii by HPLC. Bulletin of the Korean Chemical Society 30: 137-144.

Jung, H.W., J.K. Jung, K.H. Son, D.H. Lee, T.M. Kang, Y.S. Kim, and Y.K. Park. 2012. Inhibitory effects of the root extract of Dipsacus asperoides $\mathrm{C}$. Y. Cheng et al T.M.Ai on collagen-induced arthritis in mice. Journal of Ethnopharmacology 6: 98-103.

Korea Food and Drug Administration. 2007. The Korean herbal pharmacopoeia, Ministry of health family welfare of South Korea, Seoul, Korea, 216.

Korea Food and Drug Administration. 2007. The Korean herbal pharmacopoeia, Ministry of health family welfare of South Korea, The Society of Korean Official Compendium for Public Health, Seoul, Korea, 369.

Ma, X.F., T.L. Lu, C.Q. Mao, S. Song, F.Z. Yin, and L. Li. 2007. Determination of asperosaponin VI in radix Dipsaci by various processing methods with HPLC. Chinese Traditional and Herbal Drugs 38: 707-708.
Oh, S.R., K.Y. Jung, K.H. Son, S.H. Park, I.S. Lee, K.S. Ahn, and H.K. Lee. 1999. In vitro anticomplementary activity of hederagenin saponins isolated from roots of Dipsacus asper. Archives of Pharmacal Research 22: 317-319.

Pharmacopoeia Commission of People's Republic of China. 2010. Pharmacopoeia of the peoples of China, vol. 1. Beijing: The Chemical Industry Publishing House, 309.

Tomita, H., and Y. Mouri. 1996. An iridoid glucoside from Dipsacus asperoides. Phytochemistry 42: 239-240.

Wang, J.P., C.G. Zhang, X.C. Liu, and R.T. Wang. 2006. Effect of processing methods on physical and chemical properties of Radix Dipsaci. Zhong Yaocai 29: 895-897.

WHO. 2004. WHO guideline on safety monitoring of herbal medicines in pharmacovigilance systems, 1. Geneva: World Health Organization.

WHO. 2003. WHO guideline on safety monitoring of herbal medicines in pharmacovigilance systems, 17. Geneva: World Health Organization.

Zhou, Y.Q., Z.L. Yang, L. Xu, P. Li, and Y.Z. Hu. 2009. Akebia saponin D, a saponin component from Dipsacus asper Wall, protects PC 12 cells against amyloid- $\beta$ induced cytotoxicity. Cell Biology International 33: 1102-1110. 